University of Wollongong

Research Online

Faculty of Engineering - Papers (Archive)

Faculty of Engineering and Information

Sciences

$1-1-2008$

\title{
Stabilization of cobalt oxyhydrate superconductor
}

Zhi Ren

Zhejiang University

Cao Wang

Zhejiang University, caow@uow.edu.au

Xiang-fan Xu

Zhejiang University

Guang-han Cao

Zhejiang University

Zhu-an Xu

Zhejiang University

See next page for additional authors

Follow this and additional works at: https://ro.uow.edu.au/engpapers

Part of the Engineering Commons

https://ro.uow.edu.au/engpapers/5145

\section{Recommended Citation}

Ren, Zhi; Wang, Cao; Xu, Xiang-fan; Cao, Guang-han; Xu, Zhu-an; and Zhang, Yu-heng: Stabilization of cobalt oxyhydrate superconductor 2008, 2155-2157.

https://ro.uow.edu.au/engpapers/5145

Research Online is the open access institutional repository for the University of Wollongong. For further information contact the UOW Library: research-pubs@uow.edu.au 


\section{Authors}

Zhi Ren, Cao Wang, Xiang-fan Xu, Guang-han Cao, Zhu-an Xu, and Yu-heng Zhang 


\title{
Stabilization of cobalt oxyhydrate superconductor $\dagger$
}

\author{
Zhi Ren, Cao Wang, Xiang-fan Xu, Guang-han Cao,* Zhu-an Xu and Yu-heng Zhang \\ Received (in Cambridge, UK) 9th January 2008, Accepted 13th February 2008 \\ First published as an Advance Article on the web 3rd March 2008 \\ DOI: $10.1039 / b 800378$ e
}

\begin{abstract}
Stabilization of cobalt oxyhydrate superconductor was realized by post-treatment of the as-prepared superconductor in concentrated $\mathrm{NaOH}$ solution; this finding is expected to promote future experimental research on the intriguing material.
\end{abstract}

Cobalt oxyhydrate superconductor $\mathrm{Na}_{x} \mathrm{CoO}_{2} \cdot y \mathrm{H}_{2} \mathrm{O}(x \sim 0.3$, $y \sim 1.3$ ) has been studied intensively in recent years because of the unconventional superconductivity realized in a triangular lattice. ${ }^{1}$ The crystal structure consists of conducting layers of edge-sharing $\mathrm{CoO}_{6}$ octahedra pillared by $\mathrm{H}_{2} \mathrm{O} / \mathrm{Na}^{+} / \mathrm{H}_{2} \mathrm{O}$ sandwich layers. ${ }^{2,3}$ Hence, the compound is also called 'bilayer hydrate' (BLH). The BLH superconductor is unstable at ambient conditions, very easily losing parts of its crystalline water to form a monolayer hydrate (MLH) $\mathrm{Na}_{x} \mathrm{CoO}_{2} \cdot 0.6 \mathrm{H}_{2} \mathrm{O}$ whose structure is built up by identical $\mathrm{CoO}_{2}$ layers separated by mixed $\left(\mathrm{Na}^{+}, \mathrm{H}_{2} \mathrm{O}\right)$ layers. ${ }^{4-7}$ Though the formal doping level appears to be the same for both hydrates, superconductivity disappears completely in the MLH. ${ }^{4,5}$ This severe chemical instability of the BLH makes the study of the cobalt oxyhydrate superconductor very challenging. For example, during an experiment the sample handling procedure might modify the chemical composition of the superconductor to some extent. ${ }^{6}$ As a consequence, inconsistent experimental results frequently appear in the literature, which greatly limits our understanding of this 'devil superconductor'. ${ }^{8}$ To resolve this continuing controversy, it is highly desirable to explore a chemically stable superconductor in the system. ${ }^{8}$ In this communication we report the stabilization of cobalt oxyhydrate superconductor through the treatment of as-prepared superconductor in concentrated $\mathrm{NaOH}$ solution $\left(c_{\mathrm{NaOH}}=5 \mathrm{M}\right)$. The stabilized superconductor retained its BLH structure even after being annealed at $80{ }^{\circ} \mathrm{C}$ for $30 \mathrm{~min}$.

Polycrystalline samples of cobalt oxyhydrate superconductor were synthesized through the disproportionation route as described previously. ${ }^{9}$ Parent compound $\gamma-\mathrm{Na}_{x} \mathrm{CoO}_{2}$ was prepared by a solid-state reaction with high purity $\mathrm{Na}_{2} \mathrm{CO}_{3}$ and $\mathrm{Co}_{3} \mathrm{O}_{4}$ powders at $800{ }^{\circ} \mathrm{C}$ in flowing oxygen. About $1.5 \mathrm{~g}$ of $\mathrm{Na}_{x} \mathrm{CoO}_{2}$ powder was immersed in $100 \mathrm{ml}$ of $0.5 \mathrm{M} \mathrm{H}_{2} \mathrm{SO}_{4}$ solution at room temperature for $12 \mathrm{~h}$. The resultant black solid was filtered, washed with deionized water several times and then dried in air. The as-prepared BLH superconductor was obtained by hydration in a chamber at $100 \%$ relative humidity $(\mathrm{RH})$ at room temperature for one week. In the post-

Department of Physics, Zhejiang University, 38 Zheda Road, Hangzhou, People's Republic of China.E-mail: ghcao@zju.edu.cn; Fax: + 865718795 2590; Tel: + 8657187952590

$\dagger$ Electronic supplementary information (ESI) available: Details of samples' characterization. See DOI: 10.1039/b800378e treatment, about $1 \mathrm{~g}$ of the as-prepared superconductor was dispersed in $100 \mathrm{ml} 5 \mathrm{M} \mathrm{NaOH}$ solution. The mixture was magnetically stirred at room temperature for $48 \mathrm{~h}$. The final product was filtered, washed with deionized water and acetone, and then dried in air. The chemical compositions of the as-prepared and post-treated superconductors are $\mathrm{Na}_{0.20}\left(\mathrm{H}_{3} \mathrm{O}\right)_{0.15} \mathrm{CoO}_{2} \cdot 1.06 \mathrm{H}_{2} \mathrm{O}$ and $\mathrm{Na}_{0.41} \mathrm{CoO}_{2} \cdot 0.83 \mathrm{H}_{2} \mathrm{O}$, respectively. Details of the samples' characterization are provided in the ESI. $\dagger$

Fig. 1 shows the powder X-ray diffraction (XRD) patterns for the as-prepared superconductor subjected to various treatments. The as-prepared superconductor is stable at $25{ }^{\circ} \mathrm{C} 40 \%$ $\mathrm{RH}$, consisting of $\mathrm{BLH}$ single phase with lattice parameters $a=2.823 \AA$ and $c=19.77 \AA$. However, equilibration at $20 \%$ $\mathrm{RH}$ or mild thermal treatment at $40{ }^{\circ} \mathrm{C}$ for $3 \mathrm{~h}$ leads to the transformation into MLH phase with lattice parameters $a=$ $2.822 \AA$ and $c=13.80 \AA$. This result confirms that the as-prepared superconductor is sensitive to ambient humidity and temperature, in agreement with previous reports. ${ }^{4-7}$

The post-treated sample is isostructural with the as-prepared superconductor. The refined lattice parameters are $a=$ $2.827 \AA$ and $c=19.52 \AA$, consistent with the previous report. ${ }^{10}$ Compared with the as-prepared superconductor, the $a$-axis expands $\sim 0.1 \%$ while the $c$-axis shrinks by $\sim 1 \%$. The expansion of the $a$-axis reflects the decrease in Co valence; on the other hand, the remarkable shrinkage of the $c$-axis is mainly due to the removal of $\mathrm{H}_{3} \mathrm{O}^{+}$by the ion exchange of $\mathrm{Na}^{+} \cdot{ }^{10}$ Despite this subtle difference in their crystal structures, the chemical stability of the post-treated sample is noticeably higher than that of the as-prepared superconductor. The inset of Fig. 2 shows that the post-treated sample equilibrated at

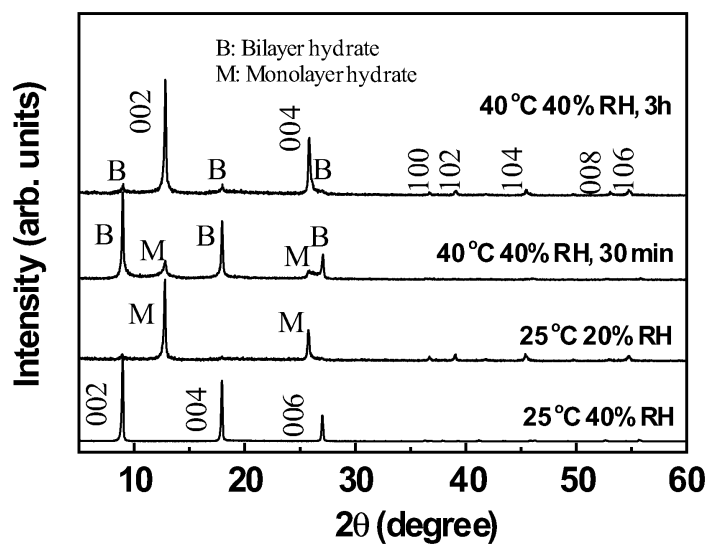

Fig. 1 Powder $\mathrm{XRD}$ patterns for the as-prepared superconductor $\mathrm{Na}_{0.20}\left(\mathrm{H}_{3} \mathrm{O}\right)_{0.15} \mathrm{CoO}_{2} \cdot 1.06 \mathrm{H}_{2} \mathrm{O}$ subjected to various treatments. 


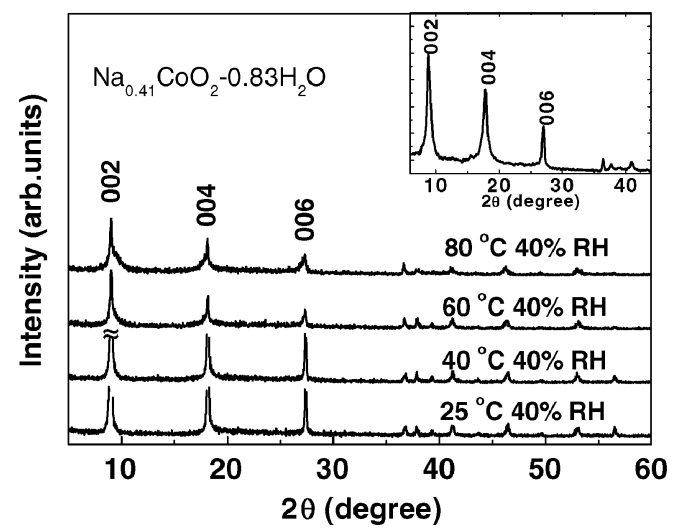

Fig. 2 XRD patterns for the post-treated sample $\mathrm{Na}_{0.41} \mathrm{CoO}_{2}$. $0.83 \mathrm{H}_{2} \mathrm{O}$ annealed at various temperatures for $30 \mathrm{~min}$ each. The inset shows the XRD pattern of the post-treated sample equilibrated at $20 \% \mathrm{RH}$ and $25^{\circ} \mathrm{C}$.

$20 \% \mathrm{RH}$ maintained the BLH structure though the diffraction peaks are slightly broadened. The BLH structure was still kept after thermal treatment up to $80^{\circ} \mathrm{C}$. Fig. 2 illustrates that there is no significant variation in the positions of the XRD peaks on heat treatment. That is to say, the lattice constant is not changed by the annealing. The $(00 l)$ diffraction peaks broaden with increasing annealing temperatures, suggesting that the crystallinity of the post-treated sample deteriorates upon heating. The deterioration of crystallinity was found to be basically irreversible, probably because the platelike crystalline grain cracks into several pieces along $a b$ planes upon heating. Nevertheless, neither MLH nor anhydrous phase was observed up to $80{ }^{\circ} \mathrm{C}$. Further increasing the annealing temperature resulted in the collapse of the BLH structure.

The thermogravimetric (TG) analysis of the as-prepared superconductor and the post-treated sample is shown in Fig. 3. For the as-prepared superconductor, the loss of water starts at a temperature as low as $40{ }^{\circ} \mathrm{C}$. The weight loss below $90{ }^{\circ} \mathrm{C}$, mainly due to the loss of crystalline water, corresponds to $\sim 50 \%$ of the total water content. In sharp contrast, the weight loss is negligible for the post-treated sample below $100^{\circ} \mathrm{C}$. The TG curve for the as-prepared superconductor equilibrated at $20 \%$ RH replicates that of the MLH reported previously. ${ }^{4,6}$ The above result clearly indicates that the post-treated BLH sample is much more stable than the as-prepared superconductor, consistent with the aforementioned XRD observations.

The chemical instability of the as-prepared cobalt oxyhydrate superconductor can be understood qualitatively as follows. The stability of a layered hydrate is determined by the interlayer ions' capability to retain their surrounding $\mathrm{H}_{2} \mathrm{O}$ molecules. ${ }^{11}$ For the as-prepared superconductor, the interlayer ions consist of $\mathrm{H}_{3} \mathrm{O}^{+}$ions, incorporated inevitably during the hydration process, ${ }^{12,13}$ in addition to $\mathrm{Na}^{+}$ions. The $\mathrm{H}_{2} \mathrm{O}$ molecules around $\mathrm{Na}^{+}$ions are retained mainly by the electrostatic interaction between the $\mathrm{Na}^{+}$ions and polar $\mathrm{H}_{2} \mathrm{O}$ molecules. In the case of the $\mathrm{H}_{3} \mathrm{O}^{+}$ions, the $\mathrm{O} \cdots \mathrm{H}-\mathrm{O}$ hydrogen bonds are responsible for keeping the surrounding $\mathrm{H}_{2} \mathrm{O}$ molecules. From an energy point of view, the hydration energy of $\mathrm{Na}^{+}$ion $\left(\sim 10^{2} \mathrm{~kJ} \mathrm{~mol}^{-1}\right)^{14}$ is one order of magni-

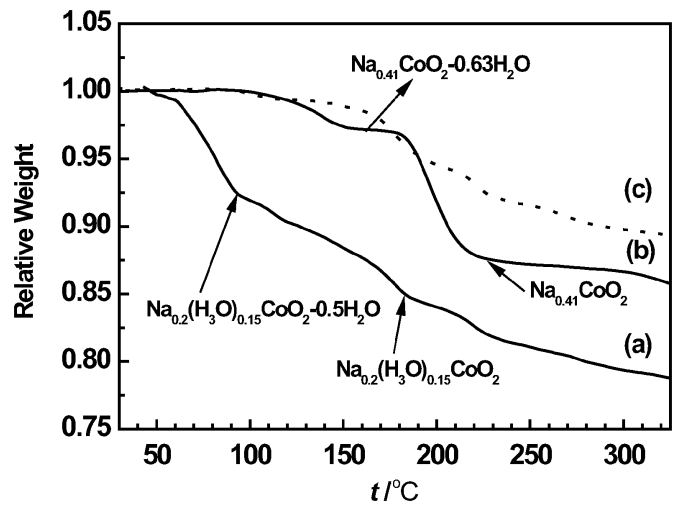

Fig. 3 Thermogravimetric curves of (a) the as-prepared superconductor, (b) the post-treated sample, and (c) the as-prepared superconductor after being equilibrated at $25{ }^{\circ} \mathrm{C}$ and $20 \% \mathrm{RH}$ (forming a MLH phase). Some intermediate products are marked in the plot.

tude larger than that of the hydronium $\left(\sim 10 \mathrm{~kJ} \mathrm{~mol}^{-1}\right) .{ }^{15} \mathrm{In}$ other words, the ability of the $\mathrm{H}_{3} \mathrm{O}^{+}$ions to grasp their surrounding $\mathrm{H}_{2} \mathrm{O}$ molecules is much lower than that of the $\mathrm{Na}^{+}$ions. Moreover, the $\mathrm{O}$ site of $\mathrm{H}_{3} \mathrm{O}^{+}$ion has a negative charge of $0.173 e$ ( $e$ is the electron charge) ${ }^{16}$ which may attract a nearby $\mathrm{Na}^{+}$ion and shield a part of its positive charge. As a consequence, the electrostatic interaction between the $\mathrm{Na}^{+}$ion and its surrounding $\mathrm{H}_{2} \mathrm{O}$ molecules is weakened in the as-prepared superconductor.

The treatment in concentrated $\mathrm{NaOH}$ solution plays a double role in stabilizing the cobalt oxyhydrate superconductor. On the one hand, incorporation of $\mathrm{H}_{3} \mathrm{O}^{+}$is greatly suppressed in the presence of concentrated $\mathrm{Na}^{+}$and $\mathrm{OH}^{-}$ ions. ${ }^{10}$ This would give rise to uniform cation layers merely consisting of $\mathrm{Na}^{+}$ions. At the same time, the $\mathrm{Na}^{+}$ions could get closer to the surrounding $\mathrm{H}_{2} \mathrm{O}$ molecules so that the electrostatic attraction between them is strengthened. ${ }^{17}$ On the other hand, chemical analysis suggests that the $\mathrm{H}_{2} \mathrm{O}$-to$\mathrm{Na}^{+}$ratio for the post-treated sample is close to $2: 1$. To achieve four $\mathrm{H}_{2} \mathrm{O}$ molecules surrounding a $\mathrm{Na}^{+}$ion, each $\mathrm{H}_{2} \mathrm{O}$ molecule may be coordinated by two neighboring $\mathrm{Na}^{+}$ions, which can be regarded as a particular case of the proposed 'Na interstitial' defects. ${ }^{3}$ In this case, the electrostatic potential that each $\mathrm{H}_{2} \mathrm{O}$ molecule feels is the sum of the contributions from two $\mathrm{Na}^{+}$ions. Hence the $\mathrm{H}_{2} \mathrm{O}$ molecules are much more difficult to lose. Combining the above two effects, the strength with which $\mathrm{Na}^{+}$ions keep their surrounding $\mathrm{H}_{2} \mathrm{O}$ molecules in the post-treated sample is greatly enhanced, resulting in a stabilized cobalt oxyhydrate superconductor.

The temperature dependence of dc magnetic susceptibility for the post-treated sample annealed under dry helium atmosphere at various temperatures is shown in Fig. 4. The posttreated sample shows bulk superconductivity with the superconducting transition temperature $T_{\mathrm{c}}=2.55 \pm 0.05 \mathrm{~K}$. We did not observe any $T_{\mathrm{c}}$ degradation after the sample had been exposed to ambient conditions for two months. Moreover, $T_{\mathrm{c}}$ remained unchanged after annealing the sample at $60{ }^{\circ} \mathrm{C}$ for $30 \mathrm{~min}$. Further increasing the annealing temperature resulted in gradual depression of $T_{\mathrm{c}}$, as shown in the inset of Fig. 4. The superconducting diamagnetism of the post-treated sample decreases with increasing annealing temperatures, which may 


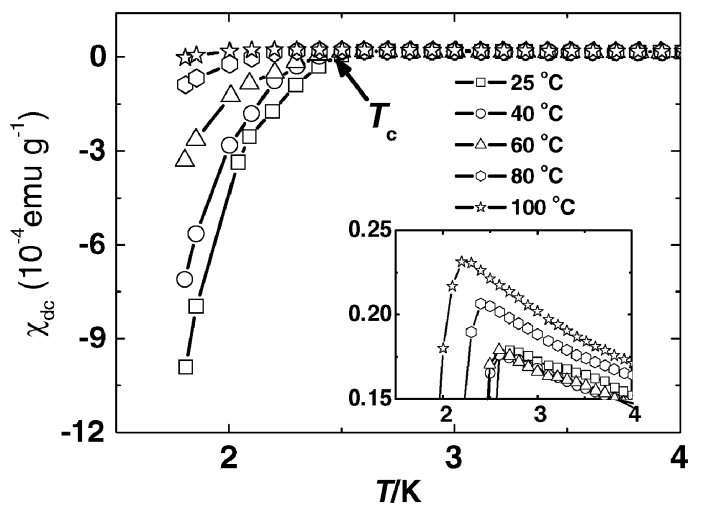

Fig. 4 Temperature dependence of dc magnetic susceptibility for the post-treated sample annealed in situ under dry helium atmosphere in a SQUID magnetometer (Quantum Design, MPMS) chamber at various temperatures for $30 \mathrm{~min}$ each. The inset shows an enlarged view of the superconducting transitions.

be attributed to the decrease of the crystallinity as indicated by the above XRD analysis.

In summary, stabilization of the cobalt oxyhydrate superconductor is feasible by the treatment of as-prepared superconductor in concentrated $\mathrm{NaOH}$ solution. The post-treated superconductor is stable under laboratory conditions $\left(25^{\circ} \mathrm{C}\right.$, $25-40 \% \mathrm{RH}$ ) for at least two months. Strikingly, it can retain the BLH structure even after annealing at $80{ }^{\circ} \mathrm{C}$ and $40 \% \mathrm{RH}$ for $30 \mathrm{~min}$. It is hoped that further study of the stabilized superconductor will resolve the continuing controversy in physical property measurements and enrich our knowledge of this fascinating material.

We acknowledge support from the National Science Foundation of China (No. 10674119), the National Basic Research Program of China (No. 2006CB601003), and the PCSIRT of the Ministry of Education of China.

\section{Notes and references}

1 (a) K. Takada, H. Sakurai, E. Takayama-Muromachi, R. A. Dilanian and T. Sasaki, Nature, 2003, 422, 53; (b) R. E. Schaak, T. Klimczuk, M. L. Foo and R. J. Cava, Nature, 2003, 424, 527; (c) G. Baskaran, Phys. Rev. Lett., 2003, 91, 097003; (d) A. Tanaka and X. Hu, Phys. Rev. Lett., 2003, 91, 257006; (e) J. V. Badding, Nat. Mater., 2003, 2, 208; (f) I. I. Mazin and M. D. Johannes, Nat. Phys., 2006, 1, 91.

2 J. W. Lynn, Q. Huang, C. M. Brown, V. L. Miller, M. L. Foo, R E. Schaak, C. Y. Jones, E. A. Mackey and R. J. Cava, Phys. Rev. B: Condens. Matter Mater. Phys., 2003, 68, 214516.

3 J. D. Jorgensen, M. Avdeev, D. G. Hinks, J. C. Burley and S. Short, Phys. Rev. B: Condens. Matter Mater. Phys., 2003, 68, 214517.

4 M. L. Foo, R. E. Schaak, V. L. Miller, T. Klimczuk, N. S. Rogado, Y. Wang, G. C. Lau, C. Cralet, H. W. Zandbergen, N. P. Ong and R. J. Cava, Solid State Commun., 2003, 127, 33.

5 K. Takada, H. Sakurai, E. Takayama-Muromachi, R. A. Dilanian and T. Sasaki, J. Solid State Chem., 2004, 177, 372.

6 V. V. Poltavets, K. A. Lokshin and M. Greenblatt, Solid State Sci., 2005, 7, 1312.

7 M. L. Foo, T. Klimczuk and R. J. Cava, Mater. Res. Bull., 2005, 40, 665.

8 R. J. Cava, Chem. Commun., 2005, 5373.

9 Z. Ren, Y. W. Wang, S. Liu, J. Wang, Z. A. Xu and G. H. Cao, Chem. Mater., 2005, 17, 1501.

10 Z. Ren, J. L. Luo, Z. A. Xu and G. H. Cao, Chem. Mater., 2007, 19, 4432.

11 J. Luo, Q. H. Zhang, A. M. Huang, O. Giraldo and S. L. Suib, Inorg. Chem., 1999, 38, 6106.

12 K. Takada, K. Fukuda, M. Osada, I. Nakai, F. Izumi, R. A Dilanian, K. Kato, M. Takata, H. Sakurai, E. TakayamaMuromachi and T. Sasaki, J. Mater. Chem., 2004, 14, 1448.

13 G. H. Cao, X. M. Tang, Y. Xu, M. Zhong, X. Z. Chen, C. M. Feng and Z. A. Xu, Solid State Commun., 2004, 131, 125.

14 Y. Marcus, Biophys. Chem., 1994, 51, 111.

15 O. Markovitch and N. Agmon, J. Phys. Chem. A, 2007, 111, 2253.

16 R. E. Kozack and P. C. Jordan, J. Chem. Phys., 1992, 96, 3131.

17 Note that the $c$-axis of the post-treated sample is $\sim 0.2 \AA$ shorter than that of the as-prepared superconductor. In contrast, the $\mathrm{CoO}_{2}$ layer thickness of the post-treated sample expands due to the reduction of the $\mathrm{Co}$ valence state. Hence the thickness of the $\mathrm{H}_{2} \mathrm{O} / \mathrm{Na}^{+} / \mathrm{H}_{2} \mathrm{O}$ intercalant layer for the post-treated sample is expected to shrink in comparison with the as-prepared superconductor. 